\title{
Measurement of Knowledge Quantity by Cognitive Time
}

\author{
Zhun $\mathrm{Cao}^{1}$
}

\author{
${ }^{1}$ Laboratory of Human-Factor and Cognitive Sciences Engineering, School of Economics and \\ Management, Southwest Jiaotong University, Chengdu 610031, China \\ 13550222811@163.com
}

Key Words: Mental Work, Knowledge Measurement, Knowledge Quantity;

\begin{abstract}
This paper describes the increasing function relationship between knowledge quantity and cognitive learning time. And constructs the measurement model differing from the current measurement principle of knowledge quantity, which possesses better validity, reliability and comparability than current knowledge quantity measurement methods. Seeks to clarify the relationship between knowledge quantity and cognitive learning time, and establish a set of effective method that is different from traditional knowledge quantity measurement on this basis.
\end{abstract}

\section{Introduction}

In the era of economic globalization today, knowledge is perceived as one of the most important assets of organizations and individuals. [1] believed that knowledge measuring is the fundamental issue for knowledge management, the focus of attention for numerous subjects as well as the most difficult problems in knowledge management [2]. However, due to the great complexity of knowledge, this thinking is unfeasible theoretically and practically and there is almost no research progress. Besides, the validity and accuracy of measuring results excessively depend on the creator of evaluation system and result estimator, so the result cannot be objective totally.

This thesis argues that knowledge is the concept of epistemology category as well as a description of motion state and state change law. Human's understanding of objective world is a learning and cognition process in time order[3]. So the author proposes a new method of measuring the knowledge quantity by adopting cognitive learning time. It's difficult for the knowledge measurement unit and standard to reach an unification; andthe comparability between measuring objects is absent, thus the effectiveness, feasibility and universality of measurement methods of knowledge quantity are lost. Therefore, the goal of the paper is to discuss the measure principles conforming to the substantive characteristics of knowledge quantity, and propose a method to construct measurement model.

\section{Is information entropy able to measure the knowledge quantity?}

Based on the fact that knowledge is also a kind of cognition about information, Shannon [4] reflected and revealed the information changing trend and law of knowledge expression through the matric characteristics of information and worked out method of measuring knowledge by principle of information measurement. Shannon's information theory is originally a "mathematical theory of communication". What communication needs to pay attention to is merely the replication of signal (the carrier of information) waveform (grammatical information) under noise background, and it's not entirely suitable for the semanticproblem of information. Knowledge quantity should be an 
absolute numerical value, which is not affected by the transmission factors or the absorption and utilization ability of the receiver (user). In other words, for a particular knowledge, even if the receiver can not understand it or be unable to absorb it, or the knowledge is even useless for the receiver, the semantic meaning carried by the knowledge still maintains the original value, and won't be reduced or become zero due to the above situation, that is to say, the contained semantic quantity is constant and the absolute quantity is unchanged.

Therefore, the information measurement principle based on uncertainty is not fully suitable for the measurement of knowledge quantity.

\section{Is the knowledge value quantity (quantity of money of knowledge products) equal to the knowledge quantity?}

There are a multitude of research literatures on the measurement of economic and social value. Scholars like Zeithaml [5], [6] discussed the measurement of knowledge as intangible assets and intellectual capital and they studied the corresponding measurement index and modes from the perspectives of economics and management. Chinese scholar Fubin [7] came to the conclusion that the value created by a person with bachelor degree is six times that created by a person with low education (junior high school and below) based on the measuring principle of knowledge value proposed by Shultz, the winner of Noble prize in economics, about "input-output".

If the knowledge quantity is measured indirectly by means of economic and social value of knowledge products, there would be difficulty in measuring with a common scale owing to the difference in form, characteristics, variety and space of the products. Economic value is merely a small part of knowledge. Knowledge value is environment-dependent and uncertain, so there are multiple values of a same knowledge depending on the means of exchange (employment, repeated transaction and one-time intellectual property business). Therefore, there is certain practical significance in indicating knowledge quantity by means of value, but it needs to combine with other measuring methods.

\section{Is it feasible to measure knowledge based on "knowledge unit/gene"?}

When the measurement of knowledge quantity faces the difficulty mentioned above, a natural thinking is to divide the knowledge to "knowledge points (cognitive unit)" [8], "knowledge unit" or "content unit" and try to measure the knowledge quantity by means of "the minimum unit which constitutes subject knowledge" ([9].

But so far there has been no way to extract a knowledge unit which can measure different or same knowledge from the vast ocean of knowledge. First, it is hard to determine the standard of knowledge unit. Second, it is hard to decompose and determine the knowledge unit [10]. Even though the two problems are solved, it is beyond imagination how to analyze, sort out and differentiate existing concepts and laws, get rid of some similar or close ones or merge and normalize them.

\section{Measuring Principle of Knowledge Quantity based on Time of Cognitive Learning}

The difficulty in measuring knowledge quantity is how to represent knowledge quantity? Knowledge quantity is the sum of knowledge of individuals in a company or a team. Studying the increase in knowledge quantity of individuals helps understand why it should be measured based on time of cognitive learning. 
Knowledge cannot exist without the subject of cognition. It grows with the cognitive development and cognition, in turn, develops with the increase of age [3]. From the perspective of ontology, time is the basic characteristic scale of object. Time quantity is the function of learning time and time is a quantity reflecting knowledge quantity or its basic characteristic scale. That is, there is a mapping relation between knowledge $\mathrm{K}$ and teaching time $\mathrm{T}: K \rightarrow T$

Moreover, according to the rough set theory, knowledge is an orderly system composed of knowledge particles with different size [11]. Also, considering that learning is a process from the easy to the difficult and complicated, knowledge can be seen as a set of ordered sequence, I, and each sequence in the set indicates a knowledge. The subscript of each element in the sequence means the position of the element in the sequence. So there is the following definition:

During teaching, the knowledge particle, $\mathrm{u}$, is always formed on the basis of existing knowledge. Therefore, teaching time for $\mathrm{u}$ should include teaching time for the knowledge base for learning $\mathrm{u}$.

For example, teaching the concept of real number can be only conducted on the basis of learning the concepts of natural number, rational number, irrational number, positive and negative number. It should be emphasized that, there is certainly difference in teaching time $\mathrm{T}$ for different $\mathrm{u}$ among different schools, teachers and students. But the average teaching time $\mathrm{T}$ for $\mathrm{u}$ can be determined by drawing samples according to the syllabus and teaching program. Here, $\mathrm{T}$ is called the socially necessary standard teaching time.

\section{Measuring Principle of Knowledge based on Standard Teaching Time}

The precondition of measuring an object is to solve the measuring principle and unit of measurement. It is known that teaching time $T_{i}$ is necessary for transforming $\Delta K_{i}$ to $K\left[S_{i}\right]$. For example, according to the syllabus of China, six years of learning in elementary school $\left(T_{i}\right)$ is required for understanding natural number $\left(\Delta K_{i}\right)$, and nine years of learning $\left(T_{i}\right)$ is required for understanding real number $\left(\Delta K_{i}\right) . \Delta K_{i}$ is the knowledge unit or knowledge point series during teaching. According to the rough set theory, $\Delta K_{i}$ can be regarded as knowledge particles of which the size can be subdivided or roughened. But no matter it is subdivided or roughened, there is always teaching time $T_{i}$ which is corresponding to $\Delta K_{i}$ on the syllabus (teaching time like teaching period, teaching hours in a week or the starting and ending points of teaching). For any knowledge particle $\Delta K_{i}$, the $\Delta K_{i}(T)$ can be always determined according to the syllabus.

In sum, knowledge quantity of $\Delta K_{i}$ and $K\left[S_{i-1}\right]$ can be scaled with teaching time $\Delta K_{n}+K\left[S_{n-1}\right] \rightarrow T_{n}, K[S]_{n} \rightarrow T_{n}$. Hence, the measuring equation of teaching time for certain knowledge (the function relationship between knowledge quantity and socially necessary learning time, and assuming $g: Q(K[S]) \rightarrow T$ ) can be derived according to equation (1): 


$$
\begin{aligned}
& Q\left(K\left[S_{0}\right]\right)+f_{1}\left(K\left[S_{0}\right], \Delta K_{1}\right) \\
& =Q\left(K\left[S_{1}\right]\right)=g_{1}\left(T_{1}\right) \\
& Q\left(K\left[S_{1}\right]\right)+f_{2}\left(K\left[S_{1}\right], \Delta K_{2}\right) \\
& =Q\left(K\left[S_{2}\right]\right)=g_{2}\left(T_{2}\right) \cdots \cdots \\
& Q\left(K\left[S_{n-1}\right]\right)+f_{n}\left(K\left[S_{n-1}\right], \Delta K_{n}\right) \\
& \left.=Q\left(K\left[S_{n}\right]\right)\right)=g_{n}\left(T_{n}\right)
\end{aligned}
$$

The sum is:

$$
\begin{aligned}
& Q\left(K\left[S_{0}\right]\right)+\sum_{i=1}^{n} f_{i}\left(K\left[S_{i-1}\right], \Delta K_{i}\right) \\
& =Q\left(K\left[S_{n}\right]\right)=\sum_{i=1}^{n} g_{i}\left(T_{i}\right)
\end{aligned}
$$

In equations (1) and (2), $Q\left(K\left[S_{0}\right]\right)$ is an initial constant. If it is assumed to be 0 , then,

$$
\begin{aligned}
& Q\left(K\left[S_{n}\right]\right)=\sum_{i=1}^{n} g_{i}\left(K\left[S_{i-1}\right], \Delta K_{i}\right) \\
& =\sum_{i=1}^{n} g_{i}\left(K\left[S_{i-1}\right](T), \Delta K_{i}(T)\right)
\end{aligned}
$$

Here, $K\left[S_{i-1}\right](T)$ is the teaching time required for acquiring $K\left[S_{i-1}\right]$, and $\Delta K_{i}(T)$ is the teaching time required for acquiring $\Delta K_{i}$. Equation (9) indicates that knowledge quantity can be measured with teaching time.

\section{Measuring Model of Knowledge Quantity}

Assuming $S=(U, A, V, f)$ is the knowledge expression system, in which $U=\left\{u_{1}, u_{2}, \ldots, u_{n} ; n \in N\right\}$ is the non-empty finite set of objects of study, or domain of discourse, $A=\left\{a_{1}, a_{2}, \ldots, a_{m} ; m \in N\right\}$ is the non-empty finite set of attributes, $V={ }_{a_{i} \in A}^{m} V\left(a_{i}\right)$ is the range of attribute $a_{i}, f: U \times A \rightarrow V$ is an information function which gives each attribute of each object an information value, that is, $\forall a \in A, u \in U, f(a, u) \subseteq V\left(a_{i}\right)$.

Usually, $S=(U, A)$ is used to replace $S=(U, A, V, f)$. Knowledge is directly related to solving problems. Corresponding knowledge is required for solving different problems. There is a correspondence between knowledge quantity and problem amount, so S can be also knowledge which is required for solving certain problem or finishing a task and it has corresponding knowledge in the syllabus. In other words, it is explicit knowledge which is corresponding to teaching time. 
For the knowledge expression system $S=(U, A, V, g)$, there is $U=\left\{u_{i j}\right\}(i=1,2,3, \cdots, n ; j=1,2,3, \cdots, m) . \quad u_{i j}$ is the knowledge particle of the ith subject at the jth subject level. $\mathrm{T}$ is the feature set of teaching time, $T=\left\{t_{1}, t_{2}, t_{3}\right\}, T \subset A$. Here, $\mathrm{t} 1=$ teaching weeks in a year, $\mathrm{t} 2=$ teaching hours in a week, $\mathrm{t} 3=$ teaching stage. $V$ is a set in which $\mathrm{A}$ can be assigned a value. $V(T)={ }_{t_{k} \in A}^{n \times m} V\left(t_{k}\right), V\left(t_{1}\right)$ is the value assigned for teaching weeks, $V\left(t_{2}\right)$ is the value assigned for the density of class hours, and $V\left(t_{3}\right)$ is that assigned for teaching stage. $g: U \times A \rightarrow V$ is the reflection of knowledge $U$ and attribute $\mathrm{A}$ on the set $\mathrm{V}$. There is a correspondence between knowledge particle and socially necessary teaching time $u_{i j} \rightarrow V\left(t_{1}, t_{2}, t_{3}\right)=V(T)$.

For any knowledge expression system $\mathrm{S}, \forall u \in U$, and $Q(S)=g(T)$ is the knowledge quantity acquired during teaching time T. Then knowledge quantity of knowledge particles $u_{i j}$ is:

$$
Q\left(u_{i j}\right)=\sum_{i=1}^{n} \operatorname{Max}_{1 \leq j \leq m}\left\{g_{i j}(T)\right\}
$$

Here, $Q\left(u_{i j}\right)$ is knowledge quantity of $u_{i j}, g_{i j}(T)$ is the function of teaching time for knowledge particle $u_{i j}$, and $m=\operatorname{Max}\{j\}$. The subscript i means the width of $u_{i j}$ or integration of different subjects. The subscript $\mathrm{j}$ means the depth of $u_{i j}$. Equation (4) means that in a subject, knowledge is like a pyramid in which the high-level knowledge covers low-level knowledge. No high-level knowledge exists without low-level one. It should be explained specially that this research aims to suggest the measuring principle and method of knowledge quantity based on standard teaching time. The specific function relationship of $g_{i j}(T)$ will be discussed in other papers.

\section{Illustration}

How to compare knowledge quantities required to solve different problems or fulfill different tasks? Here, a detailed illustration will be given. The knowledge quantity required to bake bread and that required to design a software to solve Tower of Hanoi will be compared. Apparently, the knowledge quantity of math in the first year of college is not equal to that in the first year of elementary school. This is not a question of arithmetical progression. According to the principle of psychological-physical relationship, teaching time should manifest geometrical progression.

For the convenience of illustration, assuming $g(T)=g\left(t_{0}, t_{1}, t_{2}, t_{3}\right)=\int_{t_{0}}^{t_{3}} t_{1} \times t_{2} \times t^{2} d t$ (the meaning of symbols are the same with the previous part). According to equation (4), the knowledge quantity required to solve a problem or fulfill a task is (the unit of measurement is necessary teaching time): 


$$
\begin{aligned}
& Q\left(u_{i j}\right)=\sum_{i=1}^{n} \operatorname{Max}_{1 \leq j \leq m}\left\{g_{i j}(T)\right\} \\
& =\sum_{i=1}^{n} \operatorname{Max}_{1 \leq j \leq m}\left\{u_{i j}\left(\int_{t_{0}}^{t_{3}} t_{1} \times t_{2} \times t^{2} d t\right)\right\}
\end{aligned}
$$

First, analyze and determine the necessary knowledge required for solving the problem (the minimum knowledge quantity). Assuming the minimum knowledge quantity for baking bread is $Q\left(S_{1}\right)$. It includes the physical knowledge about temperature and regulation $u_{1}$ (it requires physical knowledge in junior high school. Physics was taught since grade 2, so t0=7; the standard teaching time is 32 teaching weeks and 3 teaching hours per week. The highest-level knowledge required is that in grade 3 , so $\mathrm{t} 1=32, \mathrm{t} 2=3, \mathrm{t} 3=9$ ). It also includes arithmetical knowledge about proportion (formula) $u_{2}$ (it requires knowledge acquired in grade 5 of elementary school. The teaching of math begins at grade 1 , so $t 0=0$; and the highest-level knowledge required is that in grade 5 , so $\mathrm{t} 1=32, \mathrm{t} 2=6, \mathrm{t} 3=5)$. According to equation (11), the knowledge quantity is:

$$
\begin{aligned}
& Q\left(S_{1}\right)=Q\left(u_{1} \cup u_{2}\right) \\
& =\sum_{i=1}^{n} \operatorname{Max}_{1 \leq j \leq m}\left\{u_{i j}\left(\int_{t_{0}}^{t_{3}} t_{1} \times t_{2} \times t^{2} d t\right)\right\} \\
& =\int_{7}^{9} 32 \times 3 \times t^{2} d t+\int_{0}^{5} 32 \times 6 \times t^{2} d t \\
& =12352+8000=20352
\end{aligned}
$$

The knowledge quantity required to edit software to solve Tower of Hanoi is $Q\left(S_{2}\right)$. It includes the knowledge about program design and artificial intelligence $u_{1}$ (it requires the knowledge about program design and artificial intelligence in the first year of college. According to equation (5), the knowledge quantity is:

$$
\begin{aligned}
& Q\left(S_{2}\right)=Q\left(u_{1} \cup u_{2}\right) \\
& =\sum_{i=1}^{n} \operatorname{Max}_{1 \leq j \leq m}\left\{u_{i j}\left(\int_{t_{0}}^{t_{3}} t_{1} \times t_{2} \times t^{2} d t\right)\right\} \\
& =\int_{12}^{13} 32 \times 6 \times t^{2} d t+\int_{0}^{13} 32 \times 6 \times t^{2} d t \\
& =30016+140608=170624
\end{aligned}
$$

So, the knowledge quantity required to edit the software is eight times that required to bake bread.

\section{Conclusion}

As proposed by the paper, the current measuring principle of knowledge quantity measurement deviates from the knowledge nature features of ontology, and damages the validity, reliability and comparability of knowledge quantity measurement. This paper argues that knowledge is the crystallization of human wisdom. From the individual perspective, the individual knowledge quantity is the ordered structure that is continuously structured with the cognitive learning time from less to more, from the shallower to the deeper. Then, the paper introduces the knowledge 
equation of BROOKES, describes the growth mechanism of knowledge quantity, and thus reveals the increasing function relation between knowledge quantity and cognitive development time according to the genetic epistemology of Piaget, namely individual knowledge quantity is the increasing function of cognitive learning time. Then, the mapping relationship between knowledge quantity and "standard social teaching time" is established. Meanwhile, based on the social standard cognitive learning time" and knowledge system, it sets up the measurement model of knowledge quantity, and explores the method that is different from traditional knowledge quantity measurement. Finally, by combining with post-based knowledge quantity, it explains the calculation examples.

The paper mainly introduces the principle and method of knowledge quantity measurement based on cognitive learning time, and the rest two main tasks need to be done. Firstly, the algorithm relationship in this paper is just a simple hypothesis for ease of illustrating the measurement principle, and the specific functional form concerning it needs to be confirmed. Secondly, it is to establish a database constructed by "knowledge point (concept) and "standard social teaching time", which will facilitate too btain the knowledge quantity of this knowledge particles.

\section{References}

[1] AF Ragab, M. and A. Arisha, Knowledge management and measurement: a critical review. Journal of Knowledge Management, 2013. 17(6): p. 873-901.

[2] Chen, M.-Y., M.-J. Huang, and Y.-C. Cheng, Measuring knowledge management performance using a competitive perspective: An empirical study. Expert Systems with Applications, 2009. 36(4): p. 8449-8459.

[3] Piaget, J. and E. Duckworth, Genetic epistemology. American Behavioral Scientist, 1970. 13(3): p. 459-480.

[4] Shannon, C.E., A mathematical theory of communication. ACM SIGMOBILE Mobile Computing and Communications Review, 2001. 5(1): p. 3-55.

[5] Zeithaml, V.A., Consumer perceptions of price, quality, and value: a means-end model and synthesis of evidence. The Journal of marketing, 1988: p. 2-22.

[6] Sveiby, K.E., The new organizational wealth: Managing and measuring intangible assets. San Francisco, 1997.

[7] Fubin, Y., The new structure wage system-Ease of Social unfair income distribution. Journal of the Central Institute of Finance, 1991(06): p. 22-27.

[8] Kalyuga, S., Knowledge elaboration: A cognitive load perspective. Learning and Instruction, 2009. 19(5): p. 402-410.

[9] Marr, B., T.J. Housel, and S.K. Nelson, Knowledge valuation analysis: Applications for organizational intellectual capital. Journal of Intellectual Capital, 2005. 6(4): p. 544-557.

[10] Kankanhalli, A. and B. Tan, Knowledge management metrics: A review and directions for future research. International Journal of Knowledge Management, 2005. 1(2): p. 20-32.

[11] Youyun, Z.R.L.C.Z., Investigation to Build a Set of Domain Knowledge Particles with Rough Set Theory. Lubrication Engineering, 2007. 3: p. 029. 